\title{
Grupos funcionais em mesoambientes com piscicultura: efeitos da eutrofização artificial na dinâmica e estrutura funcional do fitoplâncton
}

\author{
Functional groups in mesoambients with fish farming: effects of artificial \\ eutrophication on the structure and functional dynamics of phytoplankton
}

\author{
Patrícia S. Cruz ${ }^{1 *}$,Luciana G. Barbosa ${ }^{2}$, Marcelo L. Rodrigues ${ }^{3}$, Suzana M. S. Lima ${ }^{4}$, Beatriz S. O. de Ceballos ${ }^{5}$
}

RESUMO - O presente estudo objetiva identificar os grupos funcionais e as espécies descritoras presentes em mesocosmos com piscicultura. O estudo foi realizado no período de janeiro a maio de 2010, com amostragens realizadas em intervalos quinzenais na subsuperfície. As variáveis abióticas obtidas foram: temperatura, transparência da água, zona eufótica, condutividade elétrica, oxigênio dissolvido, $\mathrm{pH}, \mathrm{n}$-amoniacal, nitrato, nitrito, fósforo total, fósforo reativo solúvel. Os grupos funcionais foram agrupadas com base nas características ecológicas/adaptativas. Os resultados demonstram que as condições ambientais presente nos mesocosmos favoreceram principalmente espécies Restrategistas, que apresentam elevado MDL e adaptação às condições ambientais de corpos aquáticos do semiárido, dado seus baixos requerimentos de luz e por serem aptas a explorarem ambientes com águas misturadas. Os grupos funcionais refletiram as mudanças experimentadas pela comunidade algal, fornecendo importantes informações sobre a ecologia dos ambientes, sendo notória a presença de grupos típicos de lagos rasos eutróficos.

Palavras-chave: mesocosmos, grupos funcionais, lagos rasos, piscicultura

\begin{abstract}
This study aims to identify the functional groups and species descriptors present in mesocosms with fish farming. The study was conducted from January to May 2010, with samples collected at fortnightly intervals in the subsurface. The abiotic variables were obtained: temperature, water transparency, euphotic zone, electrical conductivity, dissolved oxygen, $\mathrm{pH}$, ammonia-N, nitrate, nitrite, total phosphorus, soluble reactive phosphorus. Functional groups were grouped based on ecological characteristics / adaptive. The results demonstrate that the environmental conditions present in the mesocosms mainly favored species R-strategists, which have high CDM and adaptation to environmental conditions of water bodies semiarid, given their low light requirements and being able to explore environments with water mixed. Os functional groups reflected changes experienced by the algal community, providing important information about the ecology of the environments, and the presence of notorious groups typical of eutrophic shallow lakes.
\end{abstract}

Keywords: mesocosms; functional groups; shallow lakes; pisciculture

\section{INTRODUÇÃO}

Atualmente, a preocupação com a conservação e recuperação de ambientes rasos é crescente, principalmente por serem estes reconhecidamente vulneráveis a interferências antropogênicas e predominantes na paisagem do semiárido nordestino. Estas perturbações podem provocar fortes alterações na fauna e flora aquática e nos padrões de qualidade da água, resultando na maioria das vezes, em uma troca de estado trófico (SONDERGAARD et al., 2003), favorecendo o florescimento de cianobactérias e a redução da transparência da água.

Modificações nas condições ambientais dos corpos d'água podem ser previstas, utilizando-se agrupamentos de espécies que respondem a tais modificações em função de suas similaridades de requerimentos, morfologia e processos funcionais. Estas respostas biológicas dão-se através de variações na composição e biomassa de grupos de espécies adaptadas às características em mudança, definidas como grupos funcionais, os quais têm caracterizado as condições ambientais recentes mais acuradamente que os grandes grupos filogenéticos (KRUK et al., 2002; WEITHOFF, 2003).

Recentemente a proposta de um esquema de classificação funcional de espécies do fitoplâncton por Reynolds (1997) e Reynolds et al., (2002) foi testada e aprovada como importante ferramenta do poder preditivo (MARINHO; HUSZAR, 2002; KRUH et al., 2002; FONSECA, 2005).

Embora estudos sobre algas e grupos funcionais em abordagem experimental sejam recentes, estudos de Romo; Villena (2005) evidenciam que estes explicam significativamente a dinâmica do fitoplâncton quando comparado aos grupamentos filogenéticos. Dessa forma o

Recebido em 10082012 Aceito em 2204 2012]

1- Mestre em Ciência e Tecnologia Ambiental, Universidade Estadual da Paraíba, UEPB Email: patriciacruz biologa@hotmail.com

${ }^{2}$ - Professora titular a Universidade Federal da Paraíba, UFPB, Departamento de Ecologia; Email lucianagbarbosa@yahoo.com

3- Professor titular da Universidade Federal da paraíba, UFPB, Departamento de Zootecnia; Email: rodrigues@cca.ufpb.br

${ }^{4-}$ Doutoranda da Universidade Federal de Campina Grande Email limasuzana@uol.com.br

${ }^{5-}$ Professora titular da Universidade Estadual da Paraíba, UEPB, Centro de Ciências Biológicas e da Saúde; Email bia.ceballos@gmail.com

Revista Verde (Mossoró - RN), v. 8, n. 2, p. 27-40, abr-jun, 2013. 
presente estudo objetiva identificar os grupos funcionais e as espécies descritoras presentes em mesocosmos com piscicultura.

\section{MATERIAIS E MÉTODOS}

O presente estudo foi desenvolvido no Setor de Piscicultura do Centro de Ciências Agrárias da Universidade Federal da Paraíba (DZ/CCA/UFPB), localizado no antigo Engenho Várzea, na cidade de Areia, microrregião do Brejo Paraibano (6 $6^{\circ} 57^{\prime} 46^{\prime}$ S, $35^{\circ} 41$ ' 31 ” O), com altitude de $618 \mathrm{~m}$.

Foram construídos 15 mesocosmos, utilizando-se sacos de polietileno transparente $(0,2 \mathrm{~mm}$ de espessura), e armações circulares de alumínio (1,0m de diâmetro), além de estruturas suporte para fixação das bóias (garrafas "pet"). A confecção das estruturas seguiu as recomendações de Arcifa; Guagnoni (2003). Os mesoambientes ficaram abertos na parte superior a $30 \mathrm{~cm}$ acima da superfície da água e o fundo $(1 \mathrm{~m}$ de profundidade), foi fechado, sem contato com o sedimento. $\mathrm{O}$ experimento foi conduzido em viveiros escavados em terreno natural medindo 10 X $50 \quad \mathrm{~m}^{2}$ e com aproximadamente $1,5 \mathrm{~m}$ de profundidade.

Depois de completado o enchimento, procedeuse a adição de nutrientes e dos peixes previamente mensurados (peso e tamanho). Foram definidos 5 tratamentos (Tabela 1) seguindo as recomendações para cultivo de tilápias (KUBITZA, 2003) e três repetições para cada tratamento,distribuídos no viveiro de forma inteiramente casualizada.

Tabela 1 - Descrição dos tratamentos nos experimentos em mesocosmos com criação de tilápias (Oreochromis niloticus)

\begin{tabular}{|c|c|}
\hline TRATAMENTO & DESCRIÇÃO \\
\hline Tratamento $\left(\mathrm{T}_{1}\right)$ & (Controle - sem adubação e sem Tilápias) \\
\hline Tratamento $2\left(\mathrm{~T}_{2}\right)$ & Sem adubação + 5 Tilápias \\
\hline Tratamento $3\left(\mathrm{~T}_{3}\right)$ & $\begin{array}{c}\text { 14,88g Superfosfato Simples }+4,96 \mathrm{~g} \text { de Sulfato de } \\
\text { Amônia + } 5 \text { Tilápias }\end{array}$ \\
\hline Tratamento $4\left(\mathrm{~T}_{4}\right)$ & $\begin{array}{c}\text { 15,32g de Superfosfato Simples }+5,11 \mathrm{~g} \text { de Sulfato de } \\
\text { Amônia }+5 \text { Tilápias }\end{array}$ \\
\hline Tratamento $5\left(\mathrm{~T}_{5}\right)$ & $\begin{array}{c}\text { 15,62g de Superfosfato Simples }+5,21 \mathrm{~g} \text { de Sulfato de } \\
\text { Amônia }+5 \text { Tilápias }\end{array}$ \\
\hline
\end{tabular}

O experimento foi desenvolvido ao longo de 5 meses (Janeiro a Maio de 2010), sendo as coletas realizadas com frequiência quinzenal na subsuperfície $(0,30 \mathrm{~m}$ de profundidade). A coleta, transporte, preservação e análises das amostras seguiram as recomendações do Standard Methods for the Examination of Water and Wastewater (APHA, 2005). Foram analisadas: temperatura, transparência do disco de Secchi, fósforo total, fósforo solúvel reativo, n-amoniacal, nitrito e nitrato.

A quantificação da comunidade fitoplanctônica foi realizada em microscópio invertido com aumento de 400X, usando-se o método de sedimentação de Utermöhl (1958), seguindo as recomendações de Lund, Kpling e Lecren (1958). Com essa metodologia os indivíduos foram enumerados em campos aleatórios e contados no mínimo 100 indivíduos da espécie mais freqüente, a fim de se obter maior nível de confiabilidade. Os resultados foram expressos em densidade (ind./ml) e calculados de acordo com fórmula descrita por Ross (1979).

O cálculo do Biovolume foi estimado baseandose em 22 modelos geométricos, cujas formas aproximaram-se à forma dos indivíduos: esferas, cilindros, cones, paralelepípedos, pirâmides, (HILLEBRAND et al., 1999).
A análise descritiva dos dados físico-químicos foram realizados pelo programa STATISTICA SAS (2003). Médias aritméticas e medianas foram usadas como medidas de tendência central, desvio padrão e o coeficiente de variação de Pearson (é a razão entre o desvio padrão e a média referentes a dados de uma mesma série) como medidas de dispersão absoluta e relativa, respectivamente. Para se estabelecer o nível de significância das variações entre os tratamentos foi aplicada a análise de variância ANOVA - one-way, usando o programa estatístico STATISTICA 7. Foram considerados como resultados estatisticamente significativos aqueles para os quais o valor de $\mathrm{p}$ foi igual ou menor que 0,05 .

A Análise de Correspondência Canônica (ACC) foi realizada utilizando as principais variáveis ambientais e biológicas obtidas a partir da ACP, evitando-se colinearidade. Os programas utilizados foram o FITOPAC (SHEPHERD, 1996) para a transformação dos dados e PC-ORD, versão 3.0 para Windows (McCUNE; MEFFORD, 1997), para a execução da análise.

\section{RESULTADOS E DISCUSSÕES}


Analisando a temperatura da água nos mesoambientes, a mesma apresentou variação dos valores, tanto temporal quanto entre os distintos tratamentos, não excedendo $\pm 5^{\circ}$. Os maiores valores de transparência foram observados nos controles $\left(T_{1}\right)$, durante todo $o$ período amostral. Foram registrados no início do experimento, valores de $0,44 \mathrm{~m}$ de transparência do disco de Secchi. Á partir do $15^{\circ}$ dia, esses ambientes apresentaram valores mais elevados (mínimo de $0,52 \mathrm{~m}$ e máximo de $0,84 \mathrm{~m})$. Nos demais ambientes $\left(\mathrm{T}_{2}, \mathrm{~T}_{3}, \mathrm{~T}_{4} \mathrm{e}\right.$
$\mathrm{T}_{5}$ ) houve redução dos valores ao longo do tempo (inicial de $40 \mathrm{~cm}$ e final de $0,12 \mathrm{~cm}$ aos 84 dias), esses baixos valores de transparência apresentaram forte relação com o aumento dos valores da C.E, relacionados com o incremento das atividades metabólicas dos peixes, a exemplo da Tilapia rendalli, Lepomis macochirus e Colossoma macropomum como evidenciado em estudos de Figueredo (2000) e também com o revolvimento do sedimento (BARBOSA et al., 2006).

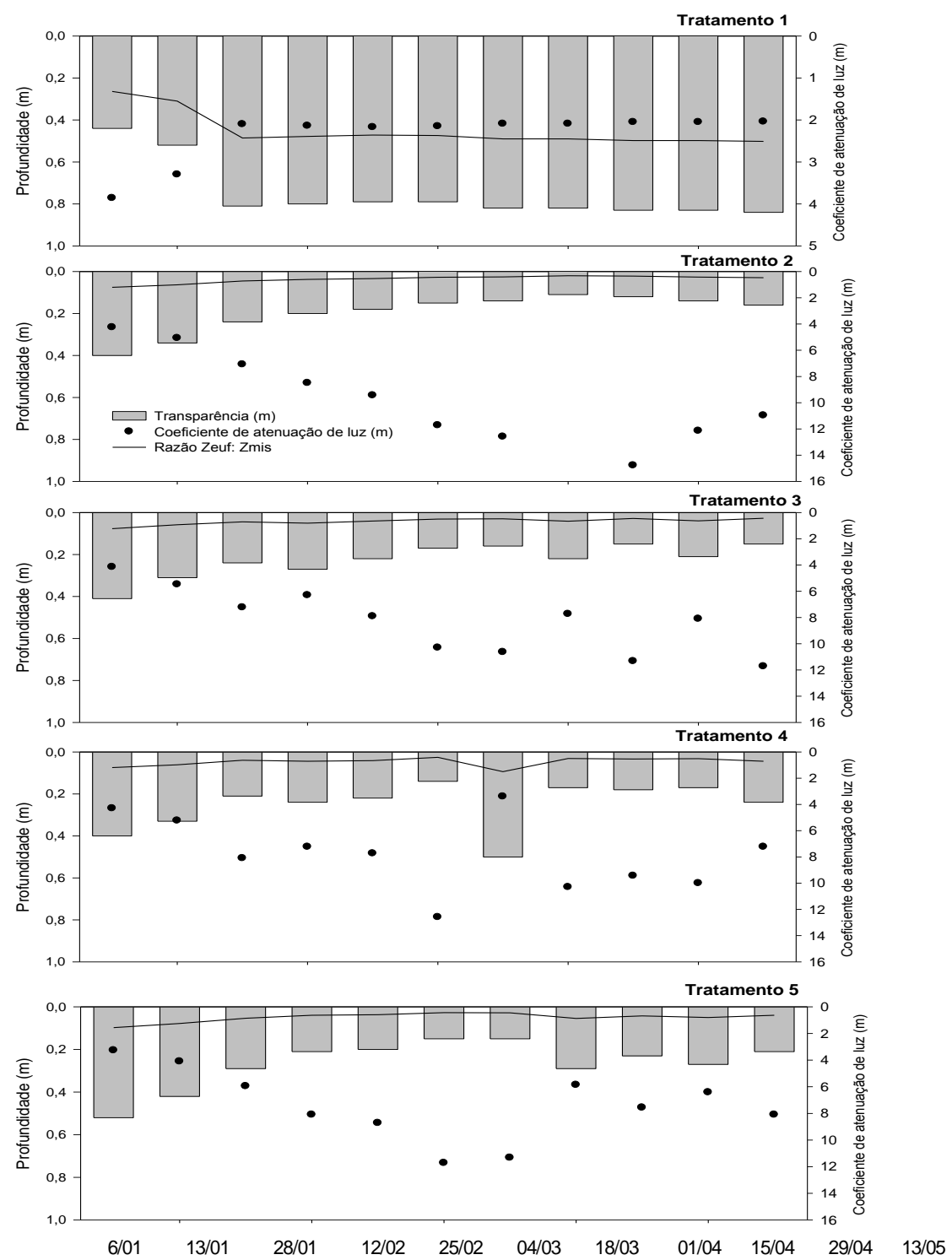

Figura1:Transparência (m) (barras), coeficiente de atenuação vertical de luz (k) (pontos) e Razão Zeuf: Zmix nos tratamentos $\left(\mathrm{T}_{1}, \mathrm{~T}_{2}, \mathrm{~T}_{3}, \mathrm{~T}_{4}\right.$ e $\left.\mathrm{T}_{5}\right)$ durante período de observação

A razão Zeu/Zmix evidenciou limitação por luz $(<1,0)$ nos ambientes com os tratamentos $\mathrm{T}_{2}, \mathrm{~T}_{3}, \mathrm{~T}_{4}$ e $\mathrm{T}_{5}$ nos meses de janeiro a maio, exceto abril. $\mathrm{O}$ coeficiente de atenuação $\left(\mathrm{K}_{0}\right)$ seguiu o mesmo perfil da Zeu/Zmix e da transparência (Figura 1). Nos mesoambientes controle $\left(\mathrm{T}_{1}\right)$ não houve limitação por luz (Zeu/Zmix > 1,0) durante 
todo o período experimental. Altos valores de atenuação de luz $\left(\mathrm{K}_{0}\right)$ foram observados em todos os tratamentos (máximo de 16,19 no tratamento $\mathrm{T}_{2}$ ), exceto no controle $\left(\mathrm{T}_{1}\right)$, onde os valores mantiveram-se abaixo de 3,86 .

Mercante et al., (2006), constatou que o fitoplâncton é a maior fonte de turbidez em viveiros de piscicultura, sendo as técnicas de manejo, como adição de ração, uma das práticas que pode potencializar o crescimento fitoplanctônico, diminuindo assim a penetração de luz no sistema. De acordo com estudos realizados por Huszar et al., (2006), em 83 reservatórios brasileiros o efeito da relação entre Zeu/Zmix mostrou que o fator limitação por luz é responsável pelas diferenças no poder preditivo de $\mathrm{N}$ e $\mathrm{P}$ em relação à biomassa fitoplanctônica.Em relação a série fosfatada, esses compostos também apresentaram diferenças significativas $(\mathrm{p}<0,0001$ e $\mathrm{F}=16,377$ ) entre os tratamentos e ao longo do período experimental. As concentrações de fósforo total no $\mathrm{T}_{2}$ registrou aumento após 4 a semana da inserção dos peixes $(>200 \mu \mathrm{g} / \mathrm{L})$ (Figura 2a). Nos demais tratamentos as concentrações foram elevadas (> $400 \mu \mathrm{g} / \mathrm{L})$ desde o início do experimento. As concentrações de PSR também foram elevadas nos tratamentos com adubação artificial e introdução dos peixes (Figura 2b).

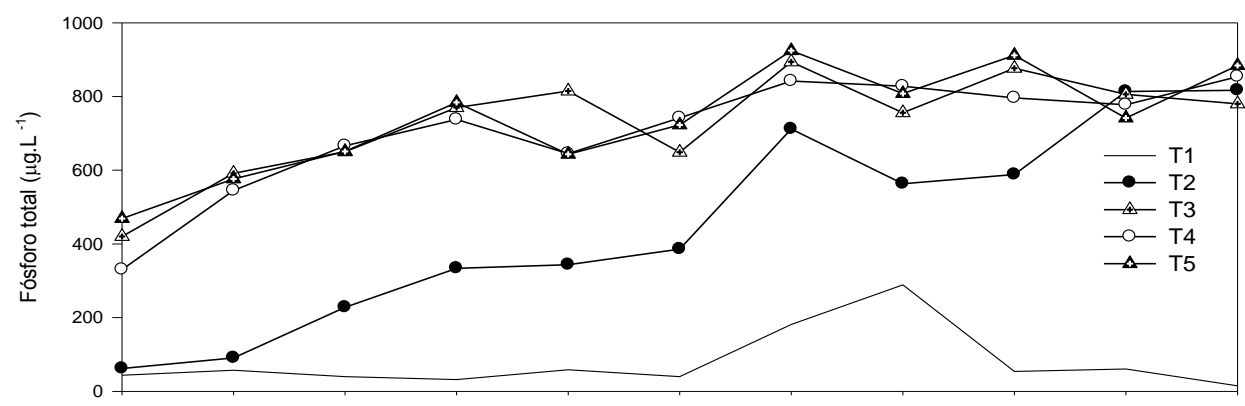

(a

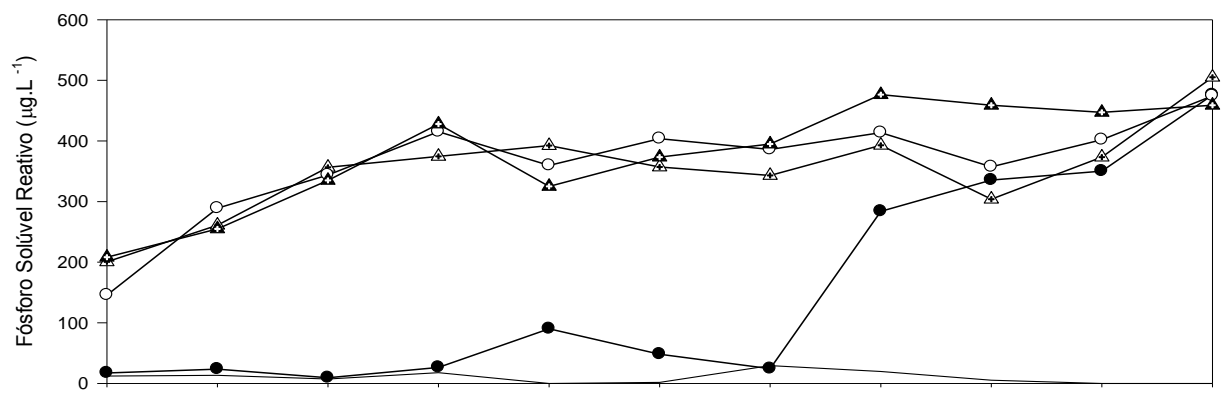

(b

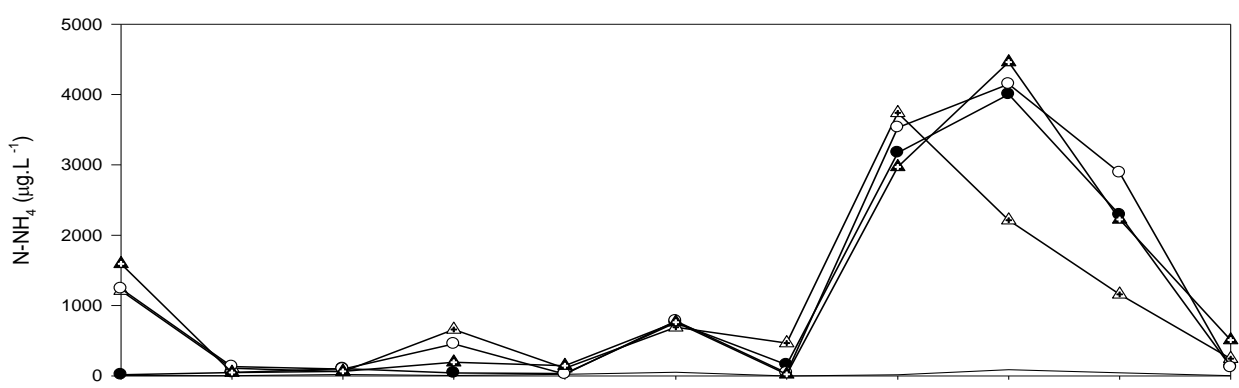

(c

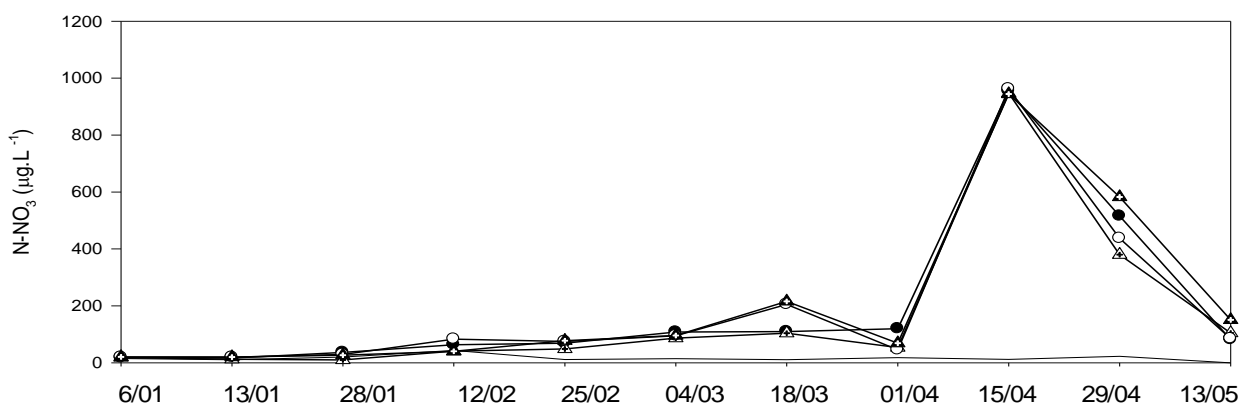

$(d$

Figura 2: Variação dos valores médios do P-total, PRS, $\mathrm{N}$-amoniacal e Nitrato nos mesoambientes $\left(\mathrm{T}_{1}, \mathrm{~T}_{2}\right.$, $\mathrm{T}_{3}, \mathrm{~T}_{4}$ e $\mathrm{T}_{5}$ ) durante período de observação 
Com exceção do controle, nos demais tratamentos as concentrações de fósforo apresentaram-se elevadas, decorrentes de diversos fatores, com destaque para o elevado aporte desse elemento advindo das fertilizações, do arraçoamento, do metabolismo dos peixes e da sua liberação na coluna d' água em consequência da degradação da matéria orgânica dos organismos mortos, incluindo as algas. Estudos de Kitamura et al., (1999) destacam o revolvimento do sedimento pelos peixes, o que contribui para que estes nutrientes retornem á coluna d'água em virtude de pouca profundidade dos ambientes.

A série nitrogenada apresentou diferenças significativas $(p=0,0007$ e $F=4.931)$ entre os tratamentos. $\mathrm{O} \mathrm{N}$-amoniacal foi a forma predominante, seguida de nitrato e nitrito, cujas concentrações apresentaram-se elevadas nos ambientes adubados artificialmente com $\mathrm{N}$ e P (>1200 $\mu \mathrm{g} / \mathrm{L})$. De acordo com Apha et al., (2005), o nitrogênio é o primeiro composto produzido na degradação da matéria orgânica. Um importante efeito sobre a concentração de $\mathrm{N}$-amoniacal foi registrado nos mesoambientes com adição de peixes. De acordo com Kubitza (2003), nos sistemas de criação os fertilizantes nitrogenados (sulfato de amônia, nitrato de amônia, fosfatos e ureia) são os principais contribuintes de nitrogênio na água. Segundo Zimmo et al., (2004), níveis de amônia total $\left(\mathrm{NH}_{4}\right.$ e $\left.\mathrm{NH}_{3}\right)$ acima de $0,5 \mathrm{mg} / \mathrm{L}$ de amônia total geralmente são prejudiciais a criação de peixes, podendo levar a incapacidade de transformar a energia alimentar em ATP.

Kubitza (2000) completa que altas concentrações deste composto pode tanto prejudicar o desenvolvimento dos peixes, como aumentar a incidência de doenças e até mesmo causar a morte indireta dos mesmos por intoxicação.
Esses valores apresentaram tendência a redução á partir da 3a a 11ạ semana. Após 92 dias, houve aumento expressivo dessas concentrações, onde os mesoambientes controle $\left(\mathrm{T}_{1}\right)$ apresentaram valores de $342,00 \mu \mathrm{g} / \mathrm{L}$ e os demais tratamentos, valores superiores a $4000 \mu \mathrm{g} / \mathrm{L}$ (Figura 2c). As concentrações de nitrato aumentaram progressivamente em todos os tratamentos, exceto no controle, atingindo no $72^{\circ}$ dia, valores médios entre 103,78 e $215,72 \mu \mathrm{g} / \mathrm{L}$ e no $100^{\circ}$ dia, valores médios entre 947,39 e $962,67 \mu \mathrm{g} / \mathrm{L}$, com tendência a redução no final do experimento (Figura 2d). O nitrito se manteve em concentrações baixas apresentando aumento significativo a partir da 9a semana até o final do experimento nos ambientes com $\mathrm{T}_{2}, \mathrm{~T}_{3}, \mathrm{~T}_{4}$ e $\mathrm{T}_{5}$. As baixas concentrações de nitrito nos ambientes $T_{2}, T_{3}, T_{4}$ e $T_{5}$ podem ser resultantes das transformações da amônia em ambiente aeróbio (nitrificação) ou pela redução do nitrato em anaerobiose, ou seja, em função das condições de oxido-redução do ambiente (PEREIRA; MERCANTE, 2005).

Sabendo-se que o IET tem por finalidade classificar os corpos d'água em diferentes graus de trofia, ou seja, avaliar a qualidade da água do ambiente, quanto ao enriquecimento por nutrientes e seu efeito relacionado ao crescimento excessivo de algas, foram analisadas as concentrações de P-total, PSR, transparência do disco de Secchi e clorofila- $a$, e calculados os IET's médio nos mesoambientes.

Os resultados do estado trófico apontam que os mesoambientes controle $\left(\mathrm{T}_{1}\right)$ enquadraram-se na faixa de condições mesotróficas, nos meses de janeiro e fevereiro, enquanto que os demais tratamentos $\left(\mathrm{T}_{2}, \mathrm{~T}_{3}, \mathrm{~T}_{4}\right.$ e $\left.\mathrm{T}_{5}\right)$ registraram condições eutróficas (IETs > 55) (Figura 3). 

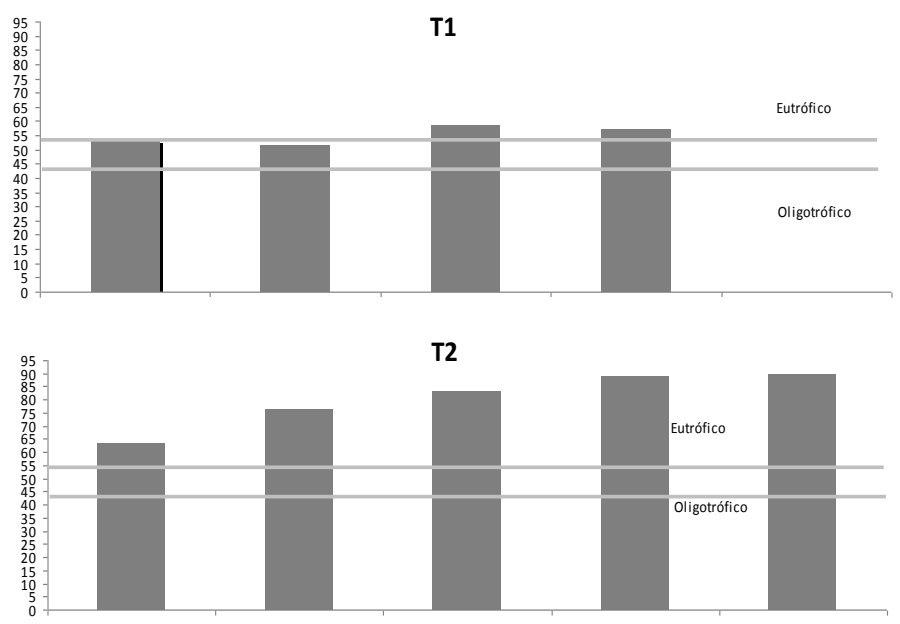

T3

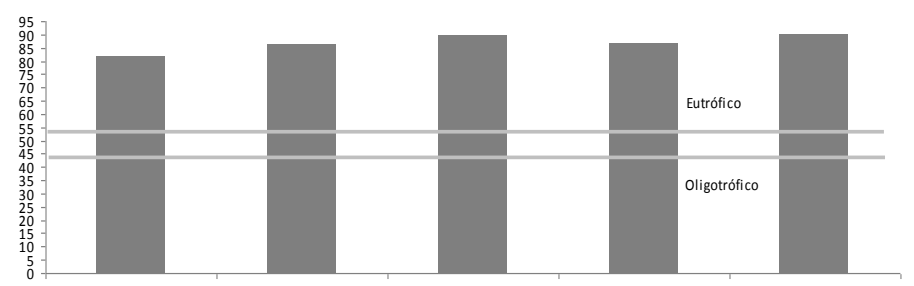

T4

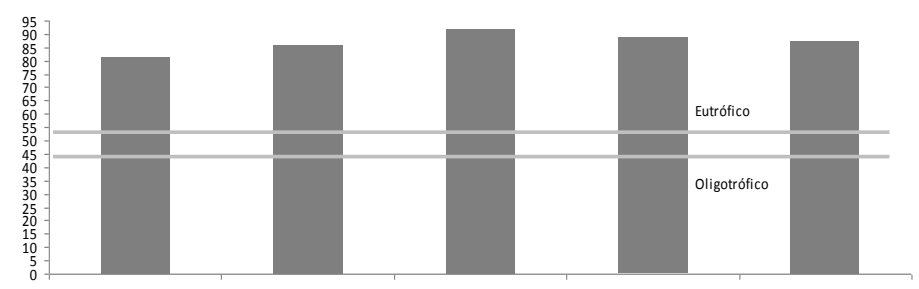

T5

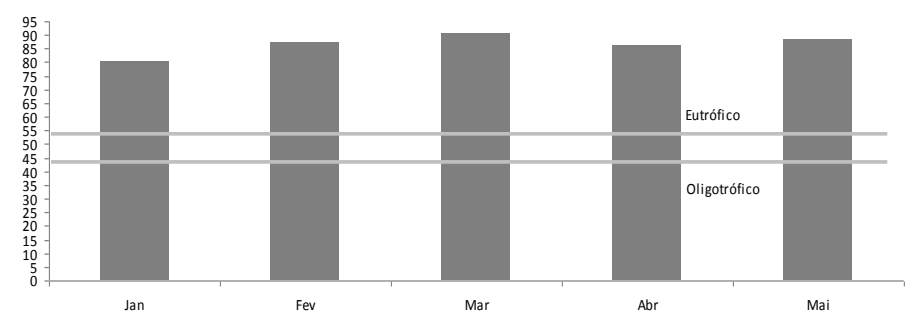

Figura 3: Valores médios dos Índices de Estado Trófico (IET's médio) nos mesoambientes $\left(\mathrm{T}_{1}, \mathrm{~T}_{2}, \mathrm{~T}_{3}, \mathrm{~T}_{4} \mathrm{e}\right.$ $\mathrm{T}_{5}$ ) durante período de observação.

Os valores dos obtidos evidenciaram ambientes eutróficos $\left(\mathrm{T}_{1}, \mathrm{~T}_{2}, \mathrm{~T}_{3}, \mathrm{~T}_{4}\right.$ e $\left.\mathrm{T}_{5}\right)$, relacionado com $\mathrm{o}$ aumento continuo de matéria orgânica ocasionado pelo aporte externo de matéria orgânica, pela senescência de elementos do plâncton e pela atividade de piscicultura. De acordo com estudos realizados por Lazarro et al., (2003) em 13 reservatórios do nordeste brasileiro, os peixes podem aumentar a turbidez da água através da ressuspensão do sedimento na procura por alimento e também estimulam as florações algais através do transporte de nutrientes do fundo para a coluna d'água e pelo consumo de zooplâncton.

O aumento expressivo da biomassa fitoplanctônica, medida como concentração de clorofila $a$, observado nos ambientes $\mathrm{T}_{2}, \mathrm{~T}_{3}, \mathrm{~T}_{4}$ e $\mathrm{T}_{5}$ ocorreu a partir da $5^{\text {a }}$ semana (valores iniciais de 32,22 e $98,02 \mu \mathrm{g} / \mathrm{L}$ e finais entre 135,97 e $188,39 \mu \mathrm{g} / \mathrm{L})$. Ao final do período experimental, as concentrações de biomassa apresentaram-se significativa e positivamente relacionadas com a concentração de fósforo total $(\mathrm{p}=0,6789$ e $\alpha=0$, 05), PSR $(\mathrm{p}=0,5223 \alpha=0,05)$ e nitrato $(\mathrm{p}=0,5505 \alpha=$ 
0,05). Essas correlações significativas evidenciadas, podem ser resultante do processo de eutrofização artificial dessas águas com os nutrientes inorgânicos, as excretas dos peixes e os restos de ração, comportamento também verificado em estudos de González (2005) e Camacho et al., (2003).
Em relação ao biovolume, a classe com maior representação foi Bacillariophyceae, com destaque para as espécies: Amphipelura lendheimerri, Fragillaria sp. e Gyrosigma kuetzengii. As espécies Closterium parvulum e Cosmarium sp., pertencentes a classe das Zygnemaphyceae registraram maiores contribuições no mês de abril (Figura4).

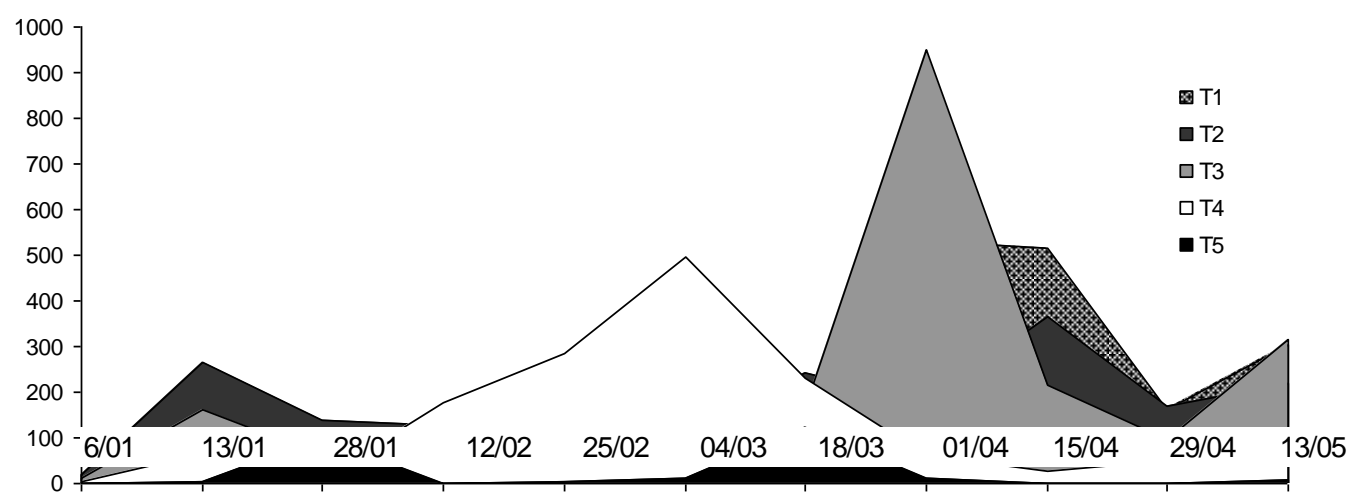

Figura4:Biovolume total da comunidade fitoplanctônica durante o período de observação

No ambiente $T_{2}$, a classe mais representativa foi a Bacillariophyceae, seguida das classes Zygnemaphyceae, Chlorophyceae e Euglenophyceae (Figura 4). De acordo com trabalhos de Gentil (2007) e Lunchi;Sipaúba-Tavares (2008), a boa expressividade dessa classe é favorecida pelas elevadas concentrações nitrogênio e fósforo.

A forte presença de Clorofíceas nos tratamentos é justificada por esse grupo ser típico de ambientes rasos eutróficos ou hipereutróficos (PADISÁK et al., 2006). O desenvolvimento da classe Euglenophyceae pode ser associado à baixa transparência da água e elevadas concentrações de matéria orgânica.

Segundo Tell; Conforti (1986), entre as microscópicas formas de vida que habitam corpos aquáticos, os euglenófitos constituem um grupo muito importante numérica e ecologicamente, especialmente em ecossistemas aquáticos que apresentam alto teor de matéria orgânica e fraco movimento de suas águas. Tal constatação é corroborada pelo comportamento heterotrófico verificado até mesmo nos representados pigmentados, que, consequentemente, podem fotossintetizar glicídios e/ou fagocitar matéria orgânica contida na coluna d'água (TELL \& CONFORTI, 1986).

As espécies com maior biovolume foram: Nizschia aciculares, Fragillaria capucina, Staurastrum leptocladum, Xantidium trilobum, Scenedesmus dimorfus, Ankitrosdesmus bernardii e Trachelomonas similis, respectivamente. Nos mesoambientes $T_{3}$, as Euglenophyceae apresentaram aumento da biomassa na segunda quinzena de março e primeira de abril, devido a contribuição da Lepocinclis sp. (Figura 5). Nos ambientes $\mathrm{T}_{4}$ e $\mathrm{T}_{5}$, as classes contribuintes foram Chlorophyceae
(Scenedesmus incrassulatus var. flexuosus) e Zygnemaphycea (Staurastrum leptocladum, Xantidium trilobum).

Em linhas gerais, as condições ambientais presente nos mesocosmos favoreceram principalmente espécies R-estrategistas, que apresentam elevado MDL (Tabela 2) e adaptação às condições ambientais de corpos aquáticos do semiárido, dado seus baixos requerimentos de luz (REYNOLDS, 1997) e por serem aptas a explorarem ambientes com águas misturadas (HAPPEYWOOD, 1988).

Os grupos observados nos mesoambientes foram: C, D, G, $\mathrm{H}_{1}, \mathrm{~J}, \mathrm{MP}, \mathrm{P}, \mathrm{S}_{1}, \mathrm{~W}_{1}$ e $\mathrm{X}_{1}$ (Tabela 2). De acordo com Weithoff (2003), a diversidade funcional reflete a multiplicidade funcional dentro de uma comunidade ao invés da multiplicidade de espécies, onde as algas com diferentes estratégias ecológicas podem estar bem adaptadas às condições ambientais similares. Nos ambientes estudados, os grupos funcionais refletiram as mudanças experimentadas pela comunidade algal, fornecendo importantes informações sobre a ecologia dos ambientes, sendo notória a presença de grupos típicos de lagos rasos eutróficos (PADISÁK; CROSSETTI; NASELLI-FLORES, 2009).

Entre os grupos funcionais identificados nos tratamentos, o grupo J (que incluiu algas verdes coloniais cenobiais), típico de ecossistemas aquáticos muito enriquecidos, rasos e misturados foi o mais relevante em biomassa em todos os tratamentos.

Nos mesoambientes $\mathrm{T}_{1}$ a baixa disponibilidade de nitrogênio favoreceu o desenvolvimento de cianobactérias heterocitadas Dolichospermum sp. De acordo com 
Sant'Anna, et al., (2006) ; Chorus ; Bartram, (1999) as cianobactérias são capazes de desenvolver estratégias que as permitem vantagens competitivas sobre determinados grupos fitoplanctônicos, tais como: capacidade de armazenamento de nitrogênio (quando este é limitante no ambiente), crescimento acelerado em períodos com temperaturas mais elevadas e presença de aerótopos que lhes permitem migrar ao longo da coluna de água (BRASIL, 2003). No entanto, apesar da representatividade pontual tais estratégias não foram suficientes para que as cianobactérias fossem dominantes nestes mesoambientes.
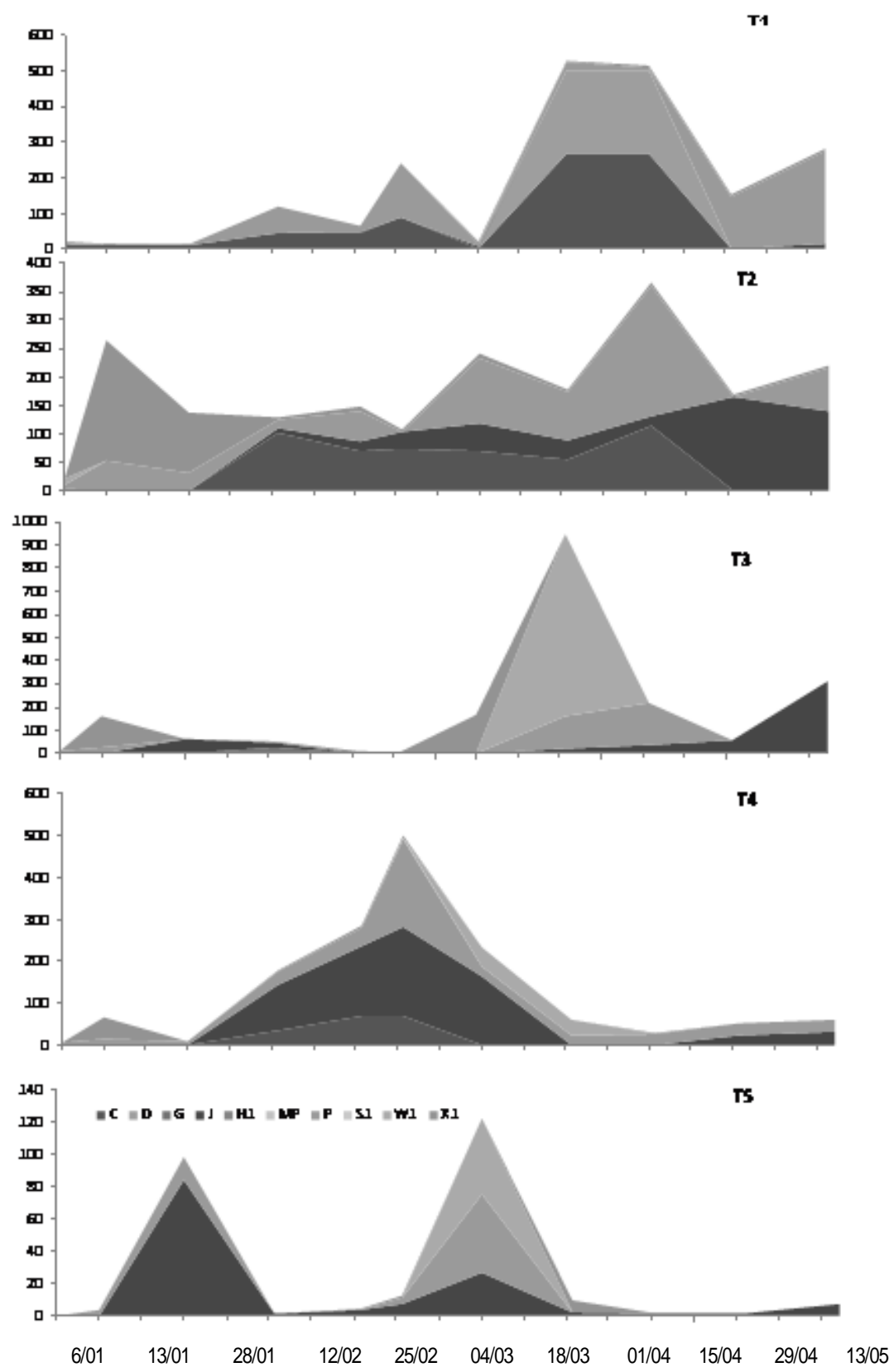

Figura 5. Biomassa dos grupos funcionais do fitoplâncton $\left(\mathrm{mg} \cdot \mathrm{L}^{-1}\right)$ durante o período de observação.

A floração de cianobactérias (Dolichospermum $s p$.) H1 em lagos rasos eutróficos é comum, pois são bem adaptadas as condições de reduzida luminosidade subaquática. A habilidade de fixar nitrogênio pelos heterócitos, além da presença de acinetos o que confere a essas espécies a capacidade de sobreviver em ecossistemas limitados por nitrogênio e com boa disponibilidade de fósforo (REYNOLDS et al., 2002), mantendo seus filamentos na camada eufótica por um longo tempo (BORGES et al., 2008).

A ocorrência da associação S1 
(cianobactéria filamentosa) pode ser justificada pelo estado de águas turbidas e deficiência de luz. Segundo Huszar et al., (2000) dominâncias de cianobactérias não fixadoras de $\mathrm{N}_{2}$, as do grupo W1, podem ser explicadas pela habilidade para auto-competirem melhor que outros grupos fitoplanctônicos por $\mathrm{NH}_{4}^{+}$.

As associações $\mathbf{C}, \mathbf{J}, \mathbf{P}$ foram as que mais contribuíram com o biovolume durante o período de estudo. Todas estas associações apresentam habilidade de se desenvolver em ecossistemas rasos e misturados. No ambiente controle $\left(\mathrm{T}_{1}\right)$, os grupos $\mathbf{C}$ e $\mathbf{P}$ apresentaram estreita relação com a transparência da água e as flutuações dos nutrientes $(\mathrm{N}$ e $\mathrm{P})$. Esse comportamento é citado por vários autores que atribuem seu sucesso a razão Zeu/Zmix $<1$ (MELO; HUSZAR, 2000; BURFORD; O' DONOHUE, 2006; NASSELLI-FLORES; BARONE, 2007).

Os resultados indicaram autovalores de 0 , 125 e 0, 054, respectivamente, para os eixos 1 e 2 . A correlação de Pearson entre ambiente e espécies para estes mesmos eixos $(0,754$ e 0,580, respectivamente) apontou as estreitas relações entre as variáveis abióticas e as espécies (Tabela 3). O Teste de Monte Carlo mostrou que a ordenação foi estatisticamente significativa $(\mathrm{p}<0,03)$ indicando a não-ocorrência de ordenação ao acaso (Figura 6).

Os coeficientes canônicos evidenciaram que a transparência $(\mathrm{r}=0,45)$ e coeficiente de atenuação da luz $(r=0,41)$, seguidas pela condutividade elétrica, razão NID:PID e fósforo solúvel reativo foram as variáveis mais importantes na ordenação do eixo 1. As correlações "intra-set" confirmaram esta tendência ao apontar fósforo solúvel reativo $(r=-0,82)$ e transparência da água $(\mathrm{r}=0,65)$. O eixo 2 apresentou coeficientes canônicos mais altos para transparência $(r=-0,61)$, coeficiente de atenuação da luz $(r=-0,53)$, fósforo solúvel reativo e condutividade elétrica $(r=-0,14)$. Para o mesmo eixo, as correlações "intra-set" apontaram condutividade elétrica $(r=-0,62)$, coeficiente de atenuação da luz $(r=-0,42)$ e fósforo solúvel reativo $(\mathrm{r}=-0,40)$ como as variáveis importantes para a ordenação.

$\mathrm{O}$ lado positivo do eixo 1 mostrou associação com as unidades amostrais do $\mathrm{T}_{1}$ (Controle), destacando-se neste caso, o grupo funcional $\mathrm{C} \quad(\mathrm{r}=1,74)$ e $\mathrm{P} \quad(0,50)$. Este agrupamento apresentou estreita relação com a transparência da água $(\mathrm{r}=0,65)$ e com as razões NID/PID ( $\mathrm{r}=-0,82)$.

O lado negativo do eixo 1 esteve associado a um agrupamento de espécies predominante nos meses de abril e maio, que se relacionou com os maiores coeficientes de atenuação da luz $(r=-0,41)$ e concentrações de Fósforo Solúvel Reativo $(\mathrm{r}=-0,82)$. Entre as unidades amostrais se destacou o grupo funcional $\mathrm{J}$ que registrou as maiores correlações $(r=-1,23)$. Os demais grupos mostraram-se associados às fases intermediárias do experimento, entre as unidades amostrais com predomínio meses de Janeiro, fevereiro e março, agrupando-se do lado positivo do eixo 2. 
Grupos funcionais em mesoambientes com piscicultura: efeitos da eutrofização artificial na dinâmica e estrutura funcional do fitoplâncton

Tabela2. Espécies descritoras do fitoplâncton (Volume, MDL e Estratégia) e grupos funcionais nos mesoambientes durante o período de observação

\begin{tabular}{|c|c|c|c|c|}
\hline Espécies & $\begin{array}{c}\text { VOLUME } \\
\text { CELULAR }\left(\mu^{3}\right)\end{array}$ & MDL $(\mu \mathrm{m})$ & ESTRATÉGIA & $\begin{array}{c}\text { GRUPO } \\
\text { FUNCIONAL }\end{array}$ \\
\hline Actinastrum hanzschii var. fluviatile & 5,04 & 3 & $\mathrm{~S}$ & $\mathrm{~J}$ \\
\hline Alaucoseira granulata & 16,49 & 21 & $\mathrm{R}$ & $P$ \\
\hline Amphipleura lendheimerii & 69,10 & 22 & $\mathrm{R}$ & $\mathrm{C}$ \\
\hline Ankistrodesmus bernadii & 885,52 & 18 & $\mathrm{~S}$ & $\mathrm{X} 1$ \\
\hline Ankistrodesmus fusiformes & 12,75 & 2 & $\mathrm{~S}$ & $\mathrm{X} 1$ \\
\hline Chlorella sp. & 65,44 & 5 & $\mathrm{C}$ & $\mathrm{X} 1$ \\
\hline Ciclotella meneguiniana & 37,69 & 4 & $\mathrm{~S}$ & $\mathrm{P}$ \\
\hline Closterium parvulum & 28,27 & 18 & $\mathrm{R}$ & $\mathrm{P}$ \\
\hline Coelastrum astroideum & 8,37 & 1 & $\mathrm{~S}$ & $\mathrm{~J}$ \\
\hline Coelastrum microporum & 16,74 & 1 & $\mathrm{~S}$ & $\mathrm{~J}$ \\
\hline Coelastrum sphaericum & 66,88 & 2 & $\mathrm{~S}$ & $\mathrm{~J}$ \\
\hline Cosmarium sp. & 282,72 & 6 & $\mathrm{~S}$ & $\mathrm{P}$ \\
\hline Dictiosphaerium pulchellum & 16,72 & 2 & $\mathrm{~S}$ & $P$ \\
\hline Dolichospermum sp. & 12,57 & 1 & $\mathrm{~S}$ & H1 \\
\hline Eudorina elegans & 98,91 & 3 & $\mathrm{~S}$ & G \\
\hline Fragillaria capucina & 50,25 & 16 & $\mathrm{R}$ & $P$ \\
\hline Fragillaria $p$. & 21,36 & 17 & $\mathrm{R}$ & $\mathrm{P}$ \\
\hline Gomphonema parvulum & 9,27 & 13 & $\mathrm{R}$ & MP \\
\hline Gomphonema sp. & 16,15 & 17 & $\mathrm{R}$ & MP \\
\hline Gyrosigma kuetzengii & 390,00 & 4 & $\mathrm{R}$ & $\mathrm{P}$ \\
\hline Gyrosigma sp. & 23,00 & 1 & $\mathrm{R}$ & $P$ \\
\hline Lepocinclis sp. & 368,60 & 11 & SR & W1 \\
\hline Monoraphidium contortum & 3,66 & 14 & $\mathrm{R}$ & $\mathrm{X} 1$ \\
\hline Monoraphidium griffthi & 3,40 & 13 & $\mathrm{R}$ & $\mathrm{X} 1$ \\
\hline Monoraphidium longiusculum & 3,14 & 12 & $\mathrm{R}$ & $\mathrm{X} 1$ \\
\hline Navicula cuspidata & 30,62 & 13 & $\mathrm{R}$ & $P$ \\
\hline Navicula nipponica & 30,62 & 13 & $\mathrm{R}$ & $\mathrm{P}$ \\
\hline Navicula sp. & 29,84 & 19 & $\mathrm{R}$ & $\mathrm{P}$ \\
\hline Nitzschia aciculares & 91,09 & 29 & $\mathrm{R}$ & $\mathrm{C}$ \\
\hline Nitzschia sp. & 80,10 & 34 & $\mathrm{R}$ & $\mathrm{D}$ \\
\hline Pediastrum tetra & 9,00 & 3 & $\mathrm{~S}$ & $\mathrm{~J}$ \\
\hline Pinnularia acrosphaeria & 690,00 & 23 & $\mathrm{R}$ & $P$ \\
\hline Pseudanabaena limnetica & 3,92 & 1 & $\mathrm{R}$ & S1 \\
\hline Scenedesmus aculeolatus & 10,47 & 5 & $\mathrm{C}$ & $\mathrm{J}$ \\
\hline Selenastrum acuminatum & 14,64 & 7 & $\mathrm{C}$ & $\mathrm{J}$ \\
\hline Scenedesmus bijugatus & 31,15 & 3 & $\mathrm{C}$ & $\mathrm{J}$ \\
\hline Scenedesmus denticulatum & 8,36 & 4 & $\mathrm{C}$ & $\mathrm{J}$ \\
\hline Scenedesmus dimorfus & 83,76 & 5 & $\mathrm{C}$ & $\mathrm{J}$ \\
\hline Scenedesmus gracile & 2,09 & 4 & $\mathrm{C}$ & $\mathrm{J}$ \\
\hline Scenedesmus incrassulatus var. flexuosus & 2010,56 & 15 & CS & $\mathrm{J}$ \\
\hline Desmodesmus intermedius & 8,36 & 4 & $\mathrm{C}$ & $\mathrm{J}$ \\
\hline Selenastrum selenastrum & 7,45 & 4 & $\mathrm{C}$ & $\mathrm{J}$ \\
\hline Staurastrum leptocladum & 35,34 & 6 & $S$ & $P$ \\
\hline Staurastrum sp. & 6,02 & 8 & $\mathrm{~S}$ & $\mathrm{P}$ \\
\hline Trachelomonas similis & 307,86 & 12 & $\mathrm{C}$ & W1 \\
\hline Xanthidium trilobum & 169,62 & 9 & $\mathrm{~S}$ & $\mathrm{P}$ \\
\hline
\end{tabular}

Revista Verde (Mossoró - RN), v. 8, n. 2, p. 27-40, abr-jun, 2013. 
Tabela 3. Coeficientes canônicos e correlações "intra-set" da Análise de Correspondência Canônica (ACC) entre variáveis ambientais e grupos funcionais dos tratamentos

\begin{tabular}{llccc}
\hline & \multicolumn{3}{c}{ Espécies descritoras } \\
\cline { 2 - 5 } \multicolumn{1}{c}{ Variável } & \multicolumn{2}{c}{ Coeficiente canônico } & \multicolumn{2}{c}{ Coeficiente de correlação } \\
& Eixo 1 & Eixo 2 & Eixo 1 & Eixo 2 \\
\cline { 2 - 5 } & $-0,190$ & $-0,140$ & $-0,53$ & $-0,62$ \\
\hline Condutividade elétrica & $-0,118$ & $-0,140$ & $-0,82$ & $-0,40$ \\
Fósforo Solúvel Reativo & 0,45 & $-0,61$ & 0,65 & 0,15 \\
Disco de Secchi & 0,41 & $-0,53$ & $-0,45$ & $-0,42$ \\
Coeficiente de atenuação de luz & 0,14 & 0,04 & 0,54 & $-0,08$ \\
Razão NID/PID & & &
\end{tabular}
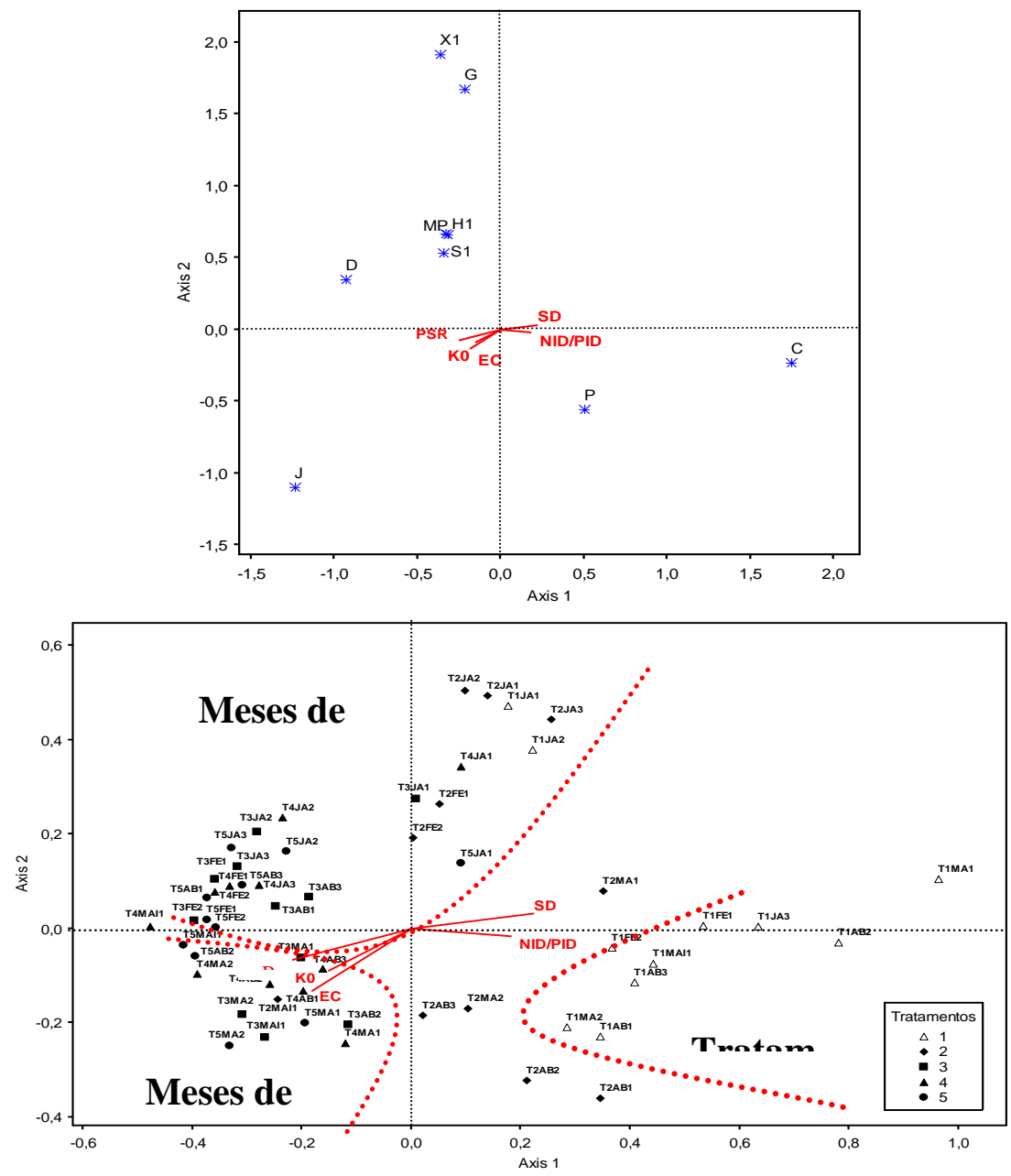

Figura 6. Diagrama de ordenação da ACC das unidades amostrais em função dos grupos funcionais (mg $\left.\mathrm{L}^{-1}\right)$ nos tratamentos e principais variáveis abióticas durante o período de observação 


\section{CONCLUSÕES}

1. Os ambientes simulados nesse estudo indicaram a vulnerabilidade de ecossistemas rasos a mudanças das águas claras para águas turbidas decorrente da ação antrópica, como a atividade de piscicultura através do elevado aporte de $\mathrm{N}$ e P.

2. A disponibilidade de luz foi um dos principais atributos relacionados à mudança na estrutura da comunidade fitoplanctônica nos ambientes estudados. Neste contexto, em ambientes naturais a ressuspensão de nutrientes pode interagir sinergicamente e antagonicamente com a sedimentação e a estabilidade da coluna d'água e determinar a magnitude e duração das florações algais.

3. As condições ambientais presente nos mesocosmos selecionaram as espécies fitoplanctônicas, de acordo com suas estratégias, com predomínio das Restrategistas em decorrência da limitação de luz presente nos tratamentos, exceção ao Controle.

4. Os grupos funcionais presentes refletiram as mudanças nas unidades experimentais, fornecendo importantes informações sobre a limnologia dos ambientes, sendo notória a presença de grupos típicos de lagos rasos eutróficos.

5. As associações verificadas nos mesoambientes foram: $\mathrm{C}, \mathrm{D}, \mathrm{G}, \mathrm{H}_{1}, \mathrm{~J}, \mathrm{MP}$, $\mathrm{P}, \mathrm{S}_{1}, \mathrm{~W}_{1}$ e $\mathrm{X}_{1}$ apresentaram estreitas relações com as variáveis abióticas. Fatores como transparência, coeficiente de atenuação da luz e razão NID/PID apresentaram grande influência na predominância dos grupos, evidenciando que o modelo das associações fitoplanctônicas funcionou muito bem nos ecossistemas rasos, simulados em mesocosmos.

\section{REFERÊNCIAS}

APHA (AMERICAM PUBLIC HEALTH ASSOCIATION). Standard methods for the examination of water and wastewater. 19 ed. Washington D.C.: APHA - AWWA - WPCF, 2005.

Arcifa, M. S. ; Guagnoni, W. A new model of enclosure for experiments in lentic water. Acta Limnol. Brasil.,15 (1): 75-79p, 2003.
Barbosa, J. E. L.; Andrade, R. S.; Lins, R. P. ; Diniz, C. R. Diagnóstico do estado trófico e aspectos limnológicos de sistemas aquáticos da Bacia Hidrográfica do Rio Taperoá, Trópico semiárido Brasileiro. Revista de Biologia e Ciências da Terra. n. $1.2^{\circ}$ semestre. p.81-89, 2006

Borges, P. A. F; Train, S.; ROdrigues, L. C.Spatial and temporal variation of phytoplankton in two subtropical Brazilian reservoirs. Hydrobiologia, v. 607, n. 1, p.63-74, 2008.

Burford, M.A.; O"Donohue, M.J. A comparison of phytoplankton community assemblages in artificially and naturally mixed subtropical water reservoirs. Freshwater Biology, Oxford, v. 51, p. 2143-2153, 2006.

BRASIL. Ministério da Saúde. FUNASA.Cianobactérias tóxicas na água para consumo humano na saúde pública e processos de remoção em água para consumo humano. Brasília: Ministério da Saúde, 2003.

Camacho, A., Wurtsbaugh, W. A., Miracle, M. R., Armengol, X. ; Vicent, E. "Nitrogen limitation of phytoplankton in a Spanish Karst lake with a deep chlorophyll maximum: a nutrient enrichment bioassay approach”. J. Plankton Res., 25 (4): 397 404, 2003.

Chorus, I. \& Bartram, J. Toxic cyanobacteria in water. A guide to their public health,consequences, monitoring and management. World Health Organization. London.416 p., 1999.

Fonseca, B.M. Diversidade fitoplanctônica como discriminador ambiental em dois reservatórios rasos com diferentes estados tróficos no Parque Estadual das Fontes do Ipiranga, São Paulo, SP. Tese de Doutorado. São Paulo: Universidade de São Paulo. 208 p., 2005.

González, M.A.S.; Jeppesen, E.; Gomá, J.; Sondergaard, M.; Jensen, J.P.; Lauridsen, T.; Lanakildehus, F. Does high nitrogen loading prevent clear - water condittions in shallow lakes at moderately high phosphorus concentrations? Freshwater Biology 50: 27-41, 2005.

Happey-Wood, C. M. Ecology of freshwater planktonic green algae. In: SANDGREN, C. D. (Ed). Growth and reproductive strategies of freshwater phytoplankton. Cambridge; Cambridge University. Pres,. ch. 5,p.175-226, 1988.

Hillebrand, H.; Dürseken, D.; KirschieL, D.; Pollingher, U.; Zohary, T. Biovolume calculation 
for pelagic and benthic microalgae. Journal of Phycology, v. 35, p. 403-424, 1999.

Huszar, V. L. M.; Silva, L. H. S.; Marinho, M.; Domingos, P. ; Sant'anna, C. L. Cyanoprokaryote assemblages in eight productive tropical Brazilian waters. Hydrobiologia, v. 424, p. 67-77, 2000.

Huszar, V.L.M.; Caraco, N.F.; Roland, F.; Cole, J. Nutrients-chlorophyll relationships in tropicalsubtropical lakes: do temperate models fit? Biogeochemistry, 2006.

Kitamura, P.C.; Silveira, M.A.; Ferráz,J.M.G.; Buschinelli, C.C.A.; CASTRO,V.L.S.S.; Chaim,A.; Corrales,F.M.; Miranda,J.I. Diagnóstico agroambiental da microbiota hidrográfica do Taquara Branca-Sumaré, SP. Jaguariúna:Embrapa-CNPMA， 25p. (EmbrapaCNPMA. Documentos, 17), 1999.

Kruk, C.; Mazzeo, N.; Lacerot, G.; Reynolds, C. S. Classification schemes for phytoplankton: A local validation of a functional approach to the analysis of species temporal replacement. Journal of Plankton Research, London, v. 24, p. 901-912, 2002.

Kubitza, F. Tilápia: tecnologia e planejamento na produção comercial. Jundiaí, SP: Fernando Kubitza, 2000. 289 p.

Kubitza, F. Qualidade da água no cultivo de camarões e peixes. Jundiaí: CPI- USP Editora, 2003.

Lazarro, X.; Bouvy, M.; Filho, R.A.R.; Oliveira, V.S.; Ssales, L.T.; VASCONCELOS, A.R.M.; Mata, M.R. Do fish regulate phytoplankton in shallow eutrophic Northeast Brazilian reservoirs? Freshwater Biology. v. 48, p.649-668, 2003.

Lunchi,G.B.; Sipaúba-Tavares,L.H. Qualidade da água fitoplanctônica de um viveiro de piscicultura utilizado para fins de pesca esportiva e irrigação. B. Inst. Pesca, 34(1):29-38p, 2008.

Lund, J.W.G.; Kpling,C. ; Lecren, E.D. The invert microscope method of stating algal number and statistical bases of estimating by counting. Hydrobiology, V11, p. 143-170, 1958.

Marinho, M. M. ; Huszar, V. L. M. Nitrogen availability and physical conditions as controllings factors of phytoplankton composition and biomass in a tropical reservoir (Southern Brazil). Archiv für Hydrobiologie, v. 153, p. 443-468, 2002.

Melo,S.; Huszar, V.L.M., Phytoplankton in an Amazonian flood-plain lake (Lago Batata, Brazil): diel variation and species strategies. J. Plankton Res. 22(1), 63-76, 2000.

Mercante, C.T.J.; Silva, D.; Costa, S.V. Avaliação da qualidade da água de pesqueiros da região metropolitana de São Paulo por meio do uso de varáveis abióticas e clorofila. In: ESTEVES, K.E.; SANT'ANNA, C.L. Pesqueiros sob uma Visão Integrada de Meio Ambiente, Saúde Pública e Manejo. São Carlos: Rima. p.37-48, 2006.

McCune, B. ; Mefford, M. J. PC-ORD. Multivariate analysis of ecological data, version 3.0. MjM Software Design, Oregon, 1997, 47 p.

Naselli-Flores, L.;Barone, R. Pluriannual morphological variability of phytoplankton in a highly productive Mediterranean reservoir (lake Arancio, Southwestern Sicily). Hydrobiologia 578, 87-95, 2007.

Padisák, J.; Grigorszky, I.; Borics, G. ; SoróczkiPintér, É. Use of phytoplankton assemblages for monitoring ecological status of lakes within the Water Framework Directive: The assemblage index. Hydrobiologia. v.553, p.1-14, 2006.

Padisák, J. Crossetti, L. O. \& Naselli-Flores, L. Use and misuse in the application of the phytoplankton functional classification: a critical review with updates. Hydrobiologia, 2009.

Pereira, L.P.F.; Mercante, C.T.J. A amônia nos sistemas de criação de peixes e seus efeitos sobre a qualidade da água. Uma revisão. B. Inst. Pesca. São Paulo, 31 (1): 81 -88, 2005.

Reynolds, C.S. Vegetation processes in the pelagic: a model for ecosystem theory. Oldendorf: Ecology Institute, 1997.

Reynolds,C.S.; Huszar, V.; Kruk,C.; NaselliFlores,L; Melo,S. Towards a functional classification of the freshwater phytoplankton. J. Plankton Res. 24: 417-428, 2002.

Romo, R., Villena, M.-J. Phytoplankton strategies and diversity under different nutrient levels and planktivorous fish densities in a shallow Mediterranean lake. J. Plankton Res. 27, 1273e1286, 2005,

Ross, J. Práticas de Ecologia. Barcelona: Ediciones Omega, 1979. p. 181

Sant'ana, C. L.; Azevedo, M. T.; Agujaro, L.; Carvalho, M. C.; Carvalho, L.; Souza, R. C. R.(2006). Manual ilustrado para identificação contagem de cianobactérias planctônicas de 
águas continetais brasileiras. Rio de Janeiro: Interciencia.

Søndegaard M., Jensen J.P. \& Jeppesen E. Role of sediment and internal loading of phosphorus in shallow lakes. Hydrobiologia, 506-509, 135-145, 2003.

Tell, G.; Conforti, V. Euglenophyta Pigmentadas de la Argentina. Stuttgart, J. Cramer. (Bibliotheca Phicologica 75), 1986.
Utermohl, H. Zur vervollkommer der quantitativen phytoplankton methodik. Mitt in Verein.theor.angew. Limnol., 9:1 - 38 p, 1958.

Weithoff,G. The concepts of 'plant funtional types' and 'funtional diversity' in lake phytoplankton- a new undertanding of phytoplankton ecology? Freshwater Biology, 48:1669-1675, 2003.

Zimmo, O. R.; Van Der Steenb, N. P.; Gijzen H. J. Nitrogem mass balance across pilot-scale algae and duckweed-based wastewater stabilization ponds, 2004. 